\title{
Orbitofrontal Epilepsy: Electroclinical Analysis of Surgical Cases and Literature Review
}

\author{
Joseph R. Smitha Karl Sillay ${ }^{c}$ Peter Winkler ${ }^{d}$ Don W. King ${ }^{b}$ \\ David W. Loring ${ }^{b}$
}

a Department of Neurosurgery, ${ }^{b}$ Department of Neurology, Medical College of Georgia, Augusta, Ga., and 'Department of Neurosurgery, Vanderbilt School of Medicine, Nashville, Tenn., USA;

dDepartment of Neurosurgery, Ludwig-Maximilians University, Munich, Germany

\section{Key Words}

Orbitofrontal epilepsy - Oroalimentary automatisms • Motor automatisms · Depth electrodes · Subdural electrodes

\begin{abstract}
Clinical and electrographic data were reviewed on 2 of our patients with orbitofrontal epilepsy who were seizure free at 5-year follow-up, and on 2 similar patients from the literature. One of our patients was lesional, and the other was nonlesional. Interictal EEG discharges were lateralized to the side of invasively recorded orbitofrontal seizures in the nonlesional case. In this case, no clinical manifestations occurred until the orbitofrontal discharge had spread to the opposite orbitofrontal and both mesial temporal areas. Unresponsiveness or arrest of activity were the initial manifestations of complex partial seizures in both cases. The 2 cases from the literature with long-term seizure-free follow-up had little impairment of awareness and displayed vigorous motor automatisms. Interictal epileptiform activity was bifrontally synchronous in 1 case. Ipsilateral frontotemporal discharges were seen in both. Invasive ictal epileptiform activity
\end{abstract}

\section{KARGER}

Fax +41613061234

E-Mail karger@karger.ch

www. karger.com
(C) 2004 S. Karger AG, Basel

1011-6125/04/0821-0020\$21.00/0

Accessible online at: www. karger.com/sfn appeared maximal in the ipsilateral orbitofrontal region in both patients. No consistent electrographic or clinical pattern characterized these 4 cases. Seizures of orbitofrontal origin may be characterized by either unresponsiveness associated with oroalimentary automatisms or limited alteration of awareness and associated with vigorous motor automatisms. Invasive monitoring of the orbitofrontal cortex should be considered in nonlesional cases with complex partial seizures that show nonlocalizing ictal patterns and interictal frontal or frontotemporal epileptiform discharges.

Copyright $@ 2004$ S. Karger AG, Basel

\section{Introduction}

There are only rare reports of orbitofrontal epilepsy documented with long-term seizure-free postoperative follow-up [1, 2]. Seizures of orbitofrontal origin have been difficult to identify electrographically and clinically. Interictally, synchronous bifrontal [3] or lateralized frontal epileptiform discharges have been associated with orbitofrontal epilepsy foci $[1,2]$, but no consistent pattern has been demonstrated. One report described ipsilateral in- 
terictal orbitofrontal epileptiform discharges using noninvasive supraorbital electrodes in a case subsequently proven to have an orbitofrontal focus with subdural recordings [1]. Also, the clinical manifestations of complex partial seizures of orbitofrontal origin have not been well defined because of the limited number of documented seizure-free cases $[2,4]$.

The primary purpose of this retrospective study and literature review was to determine if there are electrographic or clinical features that more clearly define orbitofrontal seizures.

\section{Materials and Methods}

Between 1993 and 1997, 5 patients with resections limited to the orbitofrontal cortex were identified in the series of surgical resections for intractable epilepsy at the Medical College of Georgia, Augusta, Ga., USA, and the University of Ludwig Maximilians, Munich, Germany. Ages ranged from 24 to 42 years (median age, 30 years). The median duration of epilepsy was 24 years (range, $8-39$ years).

All patients were evaluated with history and neurologic exam, neuropsychological testing (including Wada testing), and noninvasive video/EEG monitoring (including sphenoidal electrodes) [5]. MRI was performed on all patients. Ictal single photon emission computed tomography was performed in 1 case [6]. Magnetic source imaging was performed in 2 cases [7]. One patient underwent photon emission tomography scanning [8]. All electrographic data underwent initial evaluation by an epileptologist and subsequently by the epilepsy surgery team including both epileptologists and the epilepsy surgeon. The results of neuropsychological evaluation and Wada testing were initially reviewed by the neuropsychologist and then the epilepsy surgery team. MRI studies were reviewed by neuroradiology and independently by the epilepsy surgery team.

Patients undergoing invasive monitoring were implanted with either stereotactic depth [9] or subdural grid/strip electrodes [10, 11]. In all cases, at least 3 of the patient's habitual seizures were recorded with continuous video/EEG monitoring.

Intraoperative electrocorticography was performed in 4 cases. Three patients underwent intraoperative language stimulation mapping. All resections involved a combination of subpial dissection/ aspiration and en bloc resection [5]. Resections were classified as complete (or incomplete) based upon the extent of resection of the lesion, or the extent of resection of the ictal and interictal electrographic seizure focus. All resections were confined to and involved varying portions of the orbital surface of one frontal lobe.

Outcomes were classified using a slight modification of Engel's [12] criteria: seizure free (class I), rare or less than 3 seizures per year (class II), greater than $90 \%$ decease in seizure frequency (class III), and less than $90 \%$ decrease in seizures (class IV). Five-year follow-up data were available in all cases. Two patients had class I outcomes and underwent further analysis. For this analysis, we reviewed video/ EEG recordings of our 2 patients and case descriptions of the 2 patients from the literature.

Orbitofrontal Epilepsy

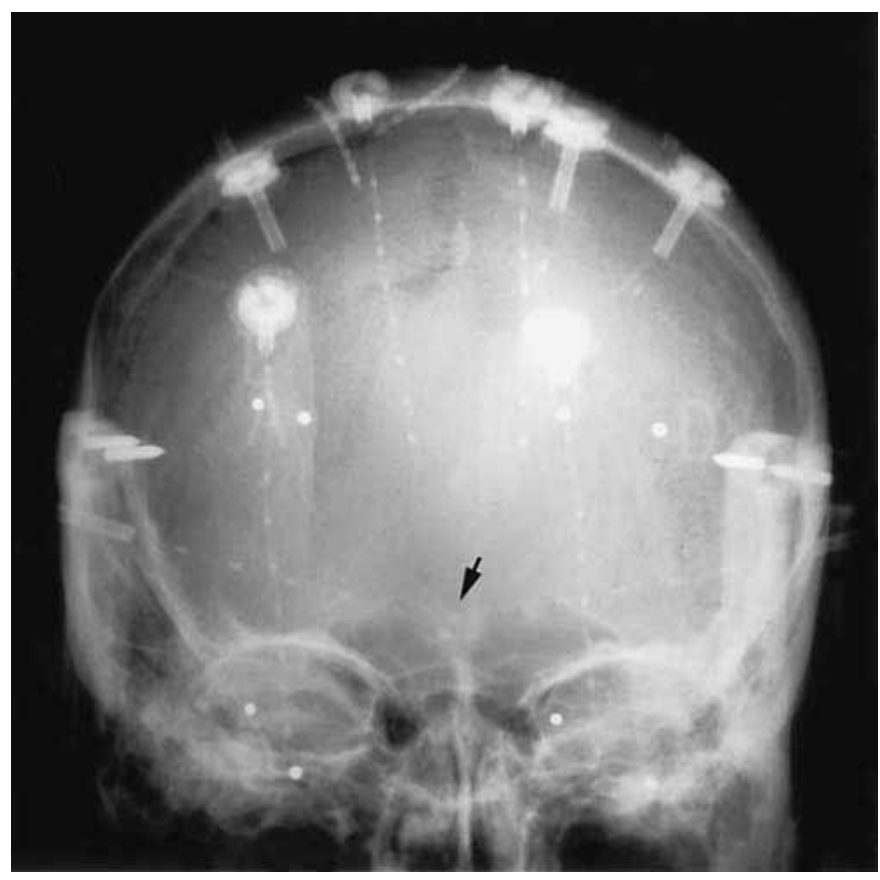

Fig. 1. The arrow points to the area of contact No. 1 of the left orbitofrontal electrode.

\section{Results}

\section{Electrographic Studies}

Of the 2 patients who were seizure free, 1 was lesional and 1 was nonlesional. The nonlesional case had interictal epileptiform discharges limited to the frontal convexity (F3), ipsilateral to the stereotactic depth electrode recorded focus. The implant is illustrated in figure 1, and the seizure recording is shown in figure 2. This electrodecremental seizure was identified independently by 2 epileptologists at the time of invasive monitoring. It should be noted that no clinical manifestations occurred until the ictal activity, which began in the left posterior orbitofrontal region, had spread to the contralateral orbitofrontal area and both mesial temporal areas. The patient did not undergo subsequent evaluation with subdural electrodes. However, our current practice would be to follow such a depth electrode evaluation with implantation of subdural electrodes to further define the epileptogenic area. The lesional case with class I outcome had no localizing interictal or ictal EEG findings.

The nonlesional class I patient had complete resection of his invasive ictal and interictal focus. This included the posterior $3.5 \mathrm{~cm}$ of the left orbitofrontal cortex (fig. 3). Histological examination revealed gliosis in the molecular 


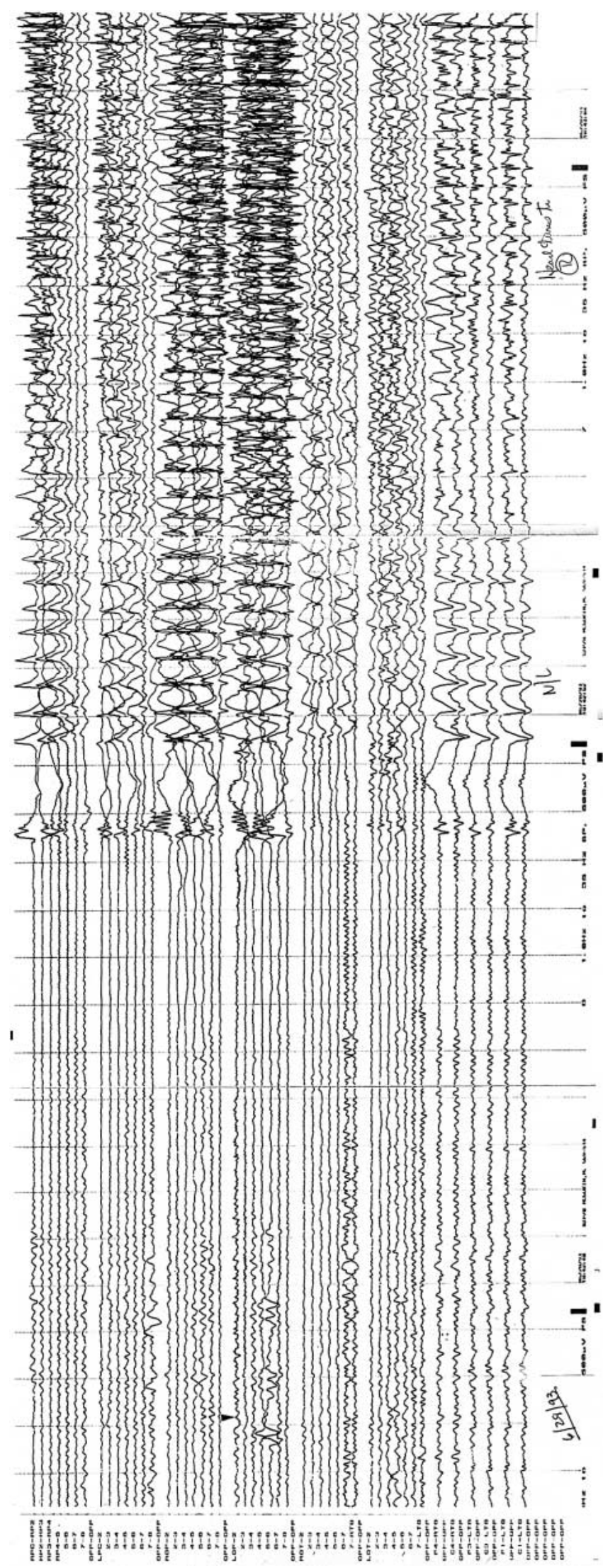

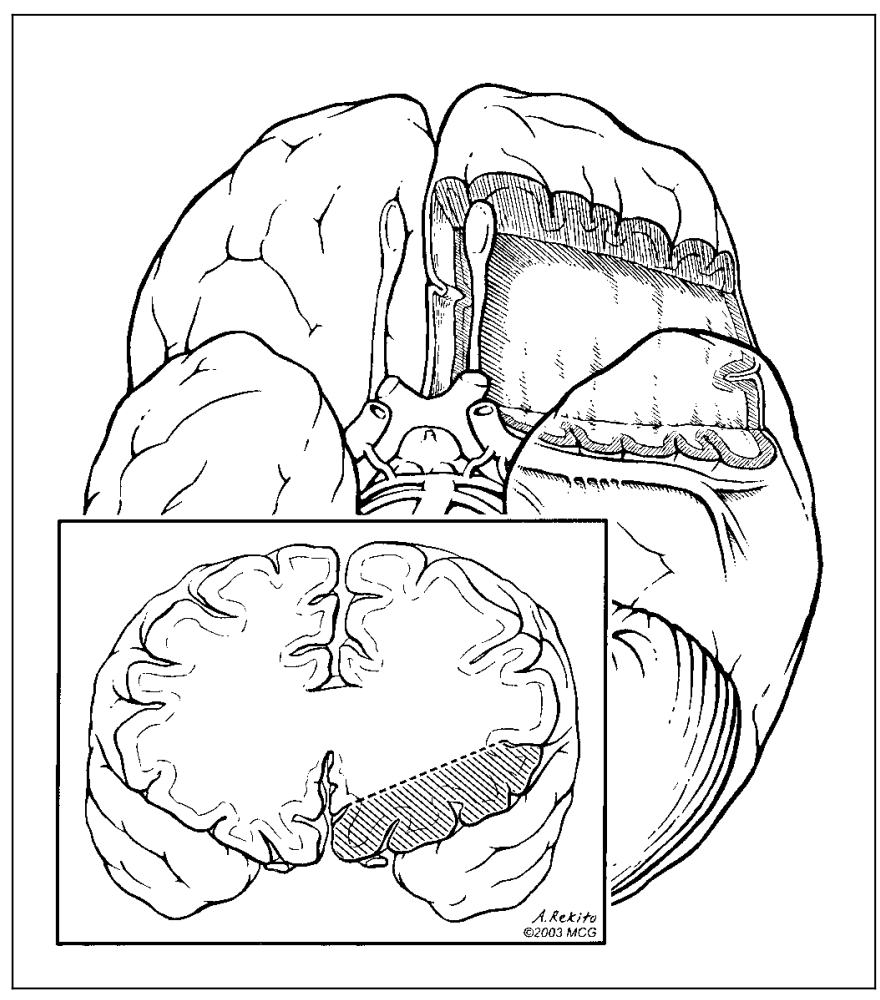

Fig. 3. Basal and coronal views show the extent of resection in our nonlesional seizure-free case. Note that the resection extended posteriorly to the trunk of the middle cerebral artery.

Fig. 2. The arrow points to the beginning of electrographic seizure in contact No. 1 of the left orbitofrontal electrode, labelled LOF. This electrodecremental seizure onset was identified independently by 2 epileptologists. Clinical seizure activity was not noted until activation of all depth electrodes, at least $16 \mathrm{~s}$ into the electrographic seizure (some time between 'N/C' and 'head turns to R'). ROF = Right orbitofrontal electrode; $\mathrm{ROT}=$ right mesial temporal electrode; $\mathrm{LOT}=$ left mesial temporal electrode; $\mathrm{RPC}=$ right mesial frontal electrode; $\mathrm{LPC}=$ left mesial frontal electrode. 
Table 1. Electrographic localization and surgical outcome

\begin{tabular}{|c|c|c|c|c|c|}
\hline Report & Noninvasive interictal EEG & $\begin{array}{l}\text { Noninvasive } \\
\text { ictal EEG }\end{array}$ & $\begin{array}{l}\text { Invasive } \\
\text { electrodes }\end{array}$ & $\begin{array}{l}\text { Invasive } \\
\text { interictal EEG }\end{array}$ & $\begin{array}{l}\text { Invasive ictal } \\
\text { EEG }\end{array}$ \\
\hline Smith et al..$^{1}$ (this study) & diffuse discharges & nonlocalized & none & none & none \\
\hline Smith et al. (this study) & F3 & nonlocalized & depth & diffuse slowing & left orbitofrontal \\
\hline Chang et al. [1] & $\begin{array}{l}\text { bisynchronous anterior discharges } \rightarrow \\
\text { right frontotemporal discharges }\end{array}$ & nonlocalized & subdural & (no data) & right orbitofrontal \\
\hline Rougier and Loiseal [2] & right frontotemporal discharges & nonlocalized & depth & (no data) & $\begin{array}{l}\text { right orbitofrontal } \\
\text { and subcallosal }\end{array}$ \\
\hline
\end{tabular}

1 Posttraumatic hematoma cavity.

Table 2. Seizure semiology of documented class I outcomes

\begin{tabular}{|c|c|c|c|c|}
\hline Author & Aura & Arrest/unresponsive & Automatisms & Vocalization \\
\hline Rougier and Loiseau [2] & none & partial & semi-purposive thrashing & shouting, laughing \\
\hline Chang et al. [1] & $\begin{array}{l}\text { body numb, flashbacks, } \\
\text { stomach butterflies, fear }\end{array}$ & usually none & pacing & yes \\
\hline \multicolumn{5}{|l|}{ Smith et al. (this study) } \\
\hline Nonlesional $^{1}$ & none & yes & none & yes \\
\hline Nonlesional $^{1}$ & metallic taste, head tingle, déjà vu & yes & none & none \\
\hline Lesional & none & yes & oromanual & none \\
\hline
\end{tabular}

1 Two seizure types.

layer and white matter with mild and patchy moderate gliosis in the other layers of the cortex. The lesional case had complete resection of the old posttraumatic hematoma cavity and surrounding sclerotic tissue.

In the case of Chang et al. [1], scalp EEG showed bisynchronous anterior epileptiform discharges, which at times extended over the right frontotemporal area. Invasive subdural monitoring showed the maximal ictal epileptiform activity in the right orbitofrontal area. In the case of Rougier and Loiseal [2], interictal discharges were noted over the right frontotemporal area with ictal activity apparently being nonlocalized. Depth electrode recordings showed the maximal ictal epileptiform activity originating in the right orbitofrontal and subcallosal areas.

Table 1 summarizes the results of noninvasive EEG, invasive EEG, and outcome following surgery in these 4 cases.

Orbitofrontal Epilepsy

\section{Seizure Semiology}

Our 2 patients displayed 3 different clinical seizure manifestations. Arrest of ongoing activity or unresponsiveness was noted initially in all seizures. This was followed by oromanual automatisms in 1 of the patients. The case of Chang et al. [1] was reported to have no impairment of responsiveness and had pacing automatisms. The case of Rougier and Loiseal [2] had incomplete impairment of responsiveness and thrashing automatisms (table 2).

Auras occurred in 2 of these 4 cases and did not seem appreciably different from cases with documented mesial temporal foci. 


\section{Discussion}

The early literature on orbitofrontal epilepsy surgery does not document any cases in which resections were confined to the orbitofrontal region, and in which longterm postoperative follow-up data were available [3, 13, 14]. More recently, 2 cases with long-term seizure-free outcomes following orbitofrontal resections have been reported [1,2].

Lateralizing frontal epileptiform discharges were present in the interictal EEG of our seizure-free nonlesional case and lateralizing frontotemporal discharges were seen in the nonlesional cases of Chang et al. [1] and Rougier and Loiseau [2]. In the case of Chang et al. [1], in which supraorbital electrodes were used, noninvasive recordings suggested an orbitofrontal focus. The presence of interictal focal frontal or bisynchronous frontal epileptiform discharges has been thought to suggest a possible orbitofrontal focus [2, 3].

Noninvasive ictal recordings were nonlocalizing in our cases and in the cases of Chang et al. [1] and Rougier and Loiseal [2]. Invasive monitoring was necessary in the cases of Chang et al. [1] and Rougier and Loiseal [2] as well as in our nonlesional case. Invasive monitoring has usually been necessary in nonlesional extratemporal cases to electrographically define the epileptogenic zone [15]. It remains controversial as to whether subdural or stereotactic depth electrodes are superior for ictal localization. It is our opinion that the orbital surface of the frontal lobe can be more completely monitored with subdural grid or strip electrodes. However, when surveying both orbitofrontal surfaces in cases with limited or no localizing or lateralizing noninvasive electrographic data, we prefer stereotactic medial and lateral orbitofrontal depth electrodes inserted through a vertex approach [9]. If seizures are lateralized to one orbitofrontal region, we subsequently monitor with subdural electrodes. Since some reported cases have shown interictal frontotemporal as well as frontal epileptiform discharges [1,2], we would include invasive monitoring of the anterior temporal area in such cases.

Munari and Bancaud [16] reported 19 cases of seizures of orbitofrontal onset documented with depth electrode recordings in 8 patients. The electrographic seizure onset preceded clinical manifestations by 4-60 s, suggesting that the initial clinical seizure manifestations were due to activation of areas outside the orbitofrontal region. The same group suggested that auras (simple partial seizures) including flashbacks, déjà vu, fear, and taste sensations are due to early spread of electrographic activity to the operculoinsulotemporoamygdalar area [17]. In our nonle- sional seizure-free case, clinical manifestations did not occur until depth electrode recordings showed bilateral orbitofrontal and anteromesial temporal electrographic spread (fig. 2). This suggests that cephalic, gustatory, and déjà vu auras (simple partial seizures) are secondary to spread of seizure activity from the orbitofrontal region to the operculoinsulotemporoamygdalar area and are of limited localizing significance. The delay of unresponsiveness (complex partial seizures) until after spread of the electrographic seizure to the contralateral orbitofrontal region and both anteromesial temporal areas also supports the view that unresponsiveness and oroalimentary automatisms are initiated outside the orbitofrontal area.

The cases of Chang et al. [1] and Rougier and Loiseal [2] had invasively recorded seizures with maximal epileptiform activity confined to the orbitofrontal and immediately adjacent frontal areas. There was no impairment of awareness in the case of Chang et al. [1] and only limited impairment of awareness in the case of Rougier and Loiseal [2]. In the case of Chang et al. [1], ipsilateral temporal subdural electrodes were present, and there did not appear to be significant spread of ictal activity to the ipsilateral temporal electrode. In our nonlesional case, which displayed alteration of awareness, there was bilateral spread of epileptiform activity before this occurred. This suggests that seizures of orbitofrontal origin without significant alteration of awareness have more limited spread of epileptiform activity.

Although seizure semiology may be helpful in localizing some seizure foci, we found no specific auras or seizure phenomena that exclusively identified the orbitofrontal area as the seizure focus. However, in our 2 seizure-free cases, complex partial seizures were characterized by unresponsiveness and oromanual automatisms. The other 2 long-term seizure-free cases we found in the literature review had seizures beginning with minimal, if any, unresponsiveness and vigorous motor automatisms. Jobst et al. [18] pointed out that focal seizures of orbitofrontal origin may resemble oroalimentary seizures of temporal lobe origin or may have explosive onsets following an aura characterized by yelling, or very vigorous motor automatisms. They characterized the latter as frontal lobe type. They reported 5 seizure-free cases (length of follow-up not stated), 2 frontal in type and 3 temporal in type. The frontal types did not have postictal amnesia.

Orbitofrontal stimulation studies have not been helpful in elucidating clinical characteristics of orbitofrontal seizures. We have stimulated 13 posterior orbitofrontal sites in 9 patients (none with orbitofrontal epilepsy) using lateral entry orbitofrontal depth electrodes (fig. 1). Pa- 
tients reported a variety of sensations, including body tingling on 6 occasions, an ill-defined smell on 1 occasion, and a cephalic sensation, light-headedness and fuzzy vision on 1 occasion each. Psychoaffective phenomena were reported on 5 occasions. These included a spacedout or confused feeling on 4 occasions and an unpleasant feeling during right-sided mesial posterior orbitofrontal stimulation on 1 occasion. An epigastric sensation was the only induced sensation we found to be associated with any of the reported seizure-free orbitofrontal seizure cases. Munari and Bancaud [16] reported olfactory hallucinations with orbitofrontal stimulation. We observed this in one instance, but postulated that this might have been related to the activation of lateral olfactory stria, since the stimulated electrode was introduced through a vertex frontal approach into the mesial posterior orbitofrontal area [9]. Recently, a patient has undergone stimulation of the lateral and mesial posterior orbitofrontal cor- tex (including the ictal zone) with stereotactic depth electrodes inserted through a vertex entry. Stimulation of up to $15 \mathrm{~mA}$ produced no clinical phenomena, supporting the hypothesis that clinical manifestations of orbitofrontal seizures may begin outside the orbitofrontal area.

\section{Conclusions}

The following behavioral and electrographic phenomena should raise the possibility of seizures of orbitofrontal origin in nonlesional cases: (1) ictally nonlocalized noninvasive EEGs with interictal frontal or frontotemporal discharges, and (2) unresponsiveness and oroalimentary automatisms or absence of unresponsiveness and vigorous motor automatisms. In such cases, invasive monitoring will be necessary to rule in or out an orbitofrontal epileptiform focus.

\section{References}

1 Chang C-N, Ojemann LM, Ojemann GA, Lettich E: Seizures of fronto-orbital origin: A proven case. Epilepsia 1991;32:487-491.

2 Rougier A, Loiseal P: Orbital frontal epilepsy: A case report. J Neurol Neurosurg Psychiatry 1988;51:146-147.

3 Penfield W, Jasper H: Epilepsy and the Functional Anatomy of the Human Brain. Boston, Little, Brown and Co, 1954.

4 Chauvel P, Kliemann F, Vignal JP, Chodkiewicz JP, Talairach $\mathrm{J}$, Bancaud $\mathrm{J}$ : The clinical signs and symptoms of frontal lobe seizures. Phenomenology and classification; in Jasper HH, Riggio S, Goldman-Rakic PS (eds): Epilepsy and the Functional Anantomy of the Frontal Lobe. New York, Raven Press, 1995 pp 115-125.

5 Flanigin HF, Smith JR: Surgical treatment of epilepsy; in Allen MB Jr, Miller RH (eds): Essentials of Neurosurgery: A Guide to Clinical Practice. New York, McGraw-Hill, 1995, pp 439-462.

6 Vera P, Habert MO, Landre E, Munari C, Chauvel P, Devaux B, Turak B, Chassoux F, Ghossoub M, Missir O: Inter-ictal brain SPET in frontal epilepsy: Correlations with stereoencephalography. Nucl Med Commun 1995; 16:591-598.
7 Smith JR, Schwartz BJ, Gallen C, Orrison W, Lewine J, Murro AM, King DW, Park YD: Utilization of multichannel magnetoencephalography in the guidance of ablative seizure surgery. J Epilepsy 1995;8:119-130.

8 Arnold S, Drzezga A, Berthel A, Werhahn KJ, Bartenstein P, Tölle TR, Weis S, Yoursry TA, Winkler PA, Noachtar S: Flumazenil- and FDG-PET reflect different aspects of epileptogenicity in extratemporal epilepsies. J Neurol 1998;255:387.

9 Smith JR, Lee MR: Stereotactic depth electrode implantation in the evaluation of candidates for ablative epilepsy surgery; in Rengachary SS, Wilkins RH (eds): Neurosurgical Operative Atlas. Park Ridge, American Association of Neurological Surgeons, 1997, pp 131-146.

10 Wyler AR: Subdural strip electrodes in surgery of epilepsy; in Lüders HO (ed): Epilepsy Surgery. New York, Raven Press, 1992, pp 395398.

11 Lesser RP, Gordon B, Fisher R, Hart J, Uematsu S: Subdural grid electrodes in surgery of epilepsy; in Lüders HO (ed): Epilepsy Surgery. New York, Raven Press, 1992, pp 399-408.

12 Engel J: Outcome with respect to epileptic seizures; in Engel J (ed): Surgical Treatment of the Epilepsies. New York, Raven Press, 1987.
13 Ludwig B, Ajmone Marsan C, Van Buren J: Cerebral seizures of probable orbitofrontal origin. Epilepsia 1975;16:141-158.

14 Tharp B: Orbital frontal seizures. An unique electroencephalographic and clinical syndrome. Epilepsia 1972;13:627-642.

15 Smith JR, Lee MR, Jenkins PD, King DW, Murro AM, Park YD, Lee GP, Meador KJ, Loring DW: A 13-year experience with epilepsy surgery. Stereotact Funct Neurosurg 1999;73: 98-103.

16 Munari C, Bancaud J: Electroclinical symptomatology of partial seizures of orbital frontal origin; in Chauvel P, Delgado-Escueta AV (eds): Frontal Lobe Seizures and Epilepsies. New York, Raven Press, 1992, pp 257-265.

17 Munari C, Tassi L, Di Leo M, Kahane P, Hoffmann D, Francione S, Quarato P: Video-stereo-electroencephalographic investigation of orbitofrontal cortex: Ictal electroclinical patterns; in Jasper HH, Riggio S, Goldman-Rakic PS (eds): Epilepsy and the Functional Anatomy of the Frontal Lobe. New York, Raven Press, 1995, pp 273-95.

18 Jobst BC, Siegel AM, Thadani VM, Roberts DW, Rhodes JC, Williamson PD: Intractable seizures of frontal origin: Clinical characteristics, localizing signs, and results of surgery. Epilepsia 2000;41:1139-1152. 